\title{
Preface: special issue of MOA 2018
}

\author{
Ya-Feng Liu ${ }^{1} \cdot$ Fengmin $\mathrm{Xu}^{2}$. Neng Fan ${ }^{3}$. Jiming Peng ${ }^{4}$ \\ Published online: 9 March 2020 \\ (c) Springer Science+Business Media, LLC, part of Springer Nature 2020
}

This special issue contains 12 selected papers from the International Workshop on Modern Optimization and Applications (MOA), which took place during June 16-18, 2018 at the Academy of Mathematics and Systems Science, Chinese Academy of Sciences, Beijing, China. A primary goal of the workshop is to bring together leading scientists, researchers, and practitioners from world to exchange and share ideas and approaches in using modern optimization techniques to model and solve real-world application problems from engineering, industry, and management. This goal was achieved to a great extent.

There are in total 18 invited speakers including Professor Sergiy Butenko (Texas A\&M University, US; Editor-in-chief of Journal of Global Optimization), Professor Oleg P. Burdakov (Linköping University, Sweden; Editor-in-Chief of Optimization Methods and Software), Professor Tsung-Hui Chang (The Chinese University of Hong Kong, Shenzhen, China), Professor Xiaojun Chen (Hong Kong Polytechnic University, HK), Professor Neng Fan (University of Arizona, US), Professor Yuantao Gu (Tsinghua University, China), Professor Zhi-Quan Luo (The Chinese University of Hong Kong, Shenzhen, China), Professor Panos M. Pardalos (University of Florida, US), Professor Jiming Peng (University of Houston, US), Professor Nick Sahinidis (Carnegie Mellon University, US; Editor-in-Chief of Optimization and Engineering), Professor Anthony Man-Cho So (The Chinese University of Hong Kong, HK), Professor Defeng Sun (Hong Kong Polytechnic University, HK), Professor Tamas Terlaky (Lehigh University, US), Professor Yanfei Wang (Institute of Geology and Geophysics, Chinese Academy of Sciences, China), Professor Zaiwen Wen (Peking University, China), Professor Hulin Wu (The University of Texas Health Science Center at

Ya-Feng Liu

yafliu@1sec.cc.ac.cn

Fengmin $\mathrm{Xu}$

fengminxu@mail.xjtu.edu.cn

Neng Fan

nfan@email.arizona.edu

Jiming Peng

jopeng@uh.edu

1 LSEC, ICMSEC, Academy of Mathematics and Systems Science, Chinese Academy of Sciences, Beijing, China

2 School of Economics and Finance, Xi' an Jiaotong University, Xi'an, China

3 Department of Systems and Industrial Engineering, University of Arizona, Tucson, USA

4 Department of Industrial Engineering, University of Houston, Houston, USA 
Houston, US), Professor Wotao Yin (University of California, Los Angeles, US), and Professor Ya-xiang Yuan (Academy of Mathematics and Systems Science, Chinese Academy of Sciences, China). In addition to the invited talks, there are also two poster sessions during the workshop. Two winners of the MOA 2018 Best Poster Award are Hailun Sun from Nanjing Normal University for the paper "Convergence analysis of sample average approximation of two-stage stochastic generalized equations" and Cong Sun from Beijing University of Posts and Telecommunications for the paper "New stepsizes for the gradient method". Over 160 participants attended the workshop. More information on the workshop can be found at the workshop website: http://lsec.cc.ac.cn/ moa2018/.

All of the 12 papers in this special issue have been peer-reviewed to the standard of the journal. The papers are from participants of the workshop and are devoted to a diverse range of topics on modern optimization and applications. For convenience of the potential readers, below we briefly summarize the contents of the papers:

- Kai Tu, Haibin Zhang, Huan Gao, and Junkai Feng propose a hybrid Bregman alternating direction method of multipliers for solving the linearly constrained difference-of-convex problems;

- Min Li, Dachuan Xu, Dongmei Zhang, and Juan Zou study seeding algorithms for spherical $k$-means clustering;

- Fengzhen Zhai and Qingna Li propose a Euclidean distance matrix (EDM) model for protein molecular conformation and develop a majorized penalty approach to solving the problem;

- Yijing Wang, Dachuan Xu, Yishui Wang, and Dongmei Zhang present four streaming algorithms for the non-submodular set function maximization problem and analyze the corresponding approximation ratios of the presented algorithms;

- Qian Liu, Yuqing Xu, and Yang Zhou propose a class of exact penalty functions and penalty algorithms for nonsmooth constrained optimization problems;

- Wanyou Cheng, Zixin Chen, and Qingjie Hu develop an active set Barzilar-Borwein algorithm for solving $\ell_{0}$ regularized optimization and show the convergence of the iterates generated by the algorithm to the strong stationary point;

- Jianlin Jiang, Su Zhang, Yibing Lv, Xin Du, and Ziwei Yan propose an ADMM-based location-allocation algorithm for solving the nonconvex constrained multi-source Weber problem under gauge and prove the convergence of the proposed algorithm;

- Fan Wu and Wei Bian propose an accelerated iterative hard thresholding algorithm for solving the $\ell_{0}$ regularized box constrained regression problem and prove the convergence of the iterates to a local minimizer of the problem;

- Min Xi, Wenyu Sun, Yannan Chen, and Hailin Sun propose a derivative-free algorithm for spherically constrained optimization and establish the global convergence of the proposed algorithm;

- Xue Gao, Xingju Cai, and Deren Han propose a Gauss-Seidel type inertial proximal alternating linearized minimization algorithm for a broad class of nonconvex and nonsmooth optimization problems;

- Minru Bai, Jing Zhao, and Zhanghui Zhang propose a descent cautious BFGS method for computing US-eigenvalues of symmetric complex tensors and establish the global convergence and superlinear convergence rate of the proposed method; and

- Florian Jarre, Felix Lieder, Ya-Feng Liu, and Cheng Lu generalize the "max-cutpolytope" in the space of real symmetric matrices with all-one diagonal to the complex case and derive set-completely positive representations of both sets. 
Finally, we thank all the authors for their interest in this special issue, and all the reviewers who helped to provide their expertise in improving the papers. We are grateful to Professor Sergiy Butenko, the Editor-in-Chief of the Journal of Global Optimization, for his great support, help, and guidance in the editorial process and Professor Yu-Hong Dai for the organization of the wonderful workshop.

Publisher's Note Springer Nature remains neutral with regard to jurisdictional claims in published maps and institutional affiliations. 